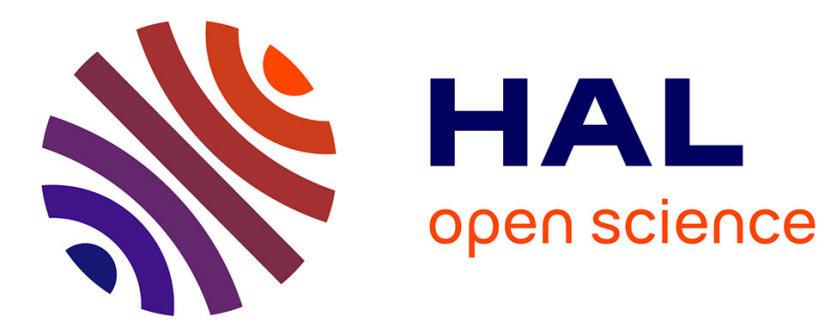

\title{
Depth extended online RPCA with spatiotemporal constraints for robust background subtraction
}

\author{
Sajid Javed, Thierry Bouwmans, Soon Ki Jung
}

\section{To cite this version:}

Sajid Javed, Thierry Bouwmans, Soon Ki Jung. Depth extended online RPCA with spatiotemporal constraints for robust background subtraction. Korea-Japan Workshop on Frontiers of Computer Vision, FCV 2015, Jan 2015, Mokpo, South Korea. pp.1 - 6, 10.1109/FCV.2015.7103745 . hal01374216

\section{HAL Id: hal-01374216 \\ https://hal.science/hal-01374216}

Submitted on 30 Sep 2016

HAL is a multi-disciplinary open access archive for the deposit and dissemination of scientific research documents, whether they are published or not. The documents may come from teaching and research institutions in France or abroad, or from public or private research centers.
L'archive ouverte pluridisciplinaire HAL, est destinée au dépôt et à la diffusion de documents scientifiques de niveau recherche, publiés ou non, émanant des établissements d'enseignement et de recherche français ou étrangers, des laboratoires publics ou privés. 


\section{Depth Extended Online RPCA with Spatiotemporal Constraints for Robust Background Subtraction}

\author{
Sajid Javed \\ School of Computer Science and \\ Engineering \\ Kyungpook National University \\ Daegu, Korea \\ Email: sajid@vr.knu.ac.kr
}

\author{
Theirry Bouwmans \\ Laboratoire MIA (Mathematiques, \\ Image et Applications) \\ Université de La Rochelle, France \\ Email: thierry.bouwmans@univ-lr.fr
}

\author{
Soon Ki Jung \\ School of Computer Science and \\ Engineering \\ Kyungpook National University \\ Daegu, Korea \\ Email: skjung@knu.ac.kr
}

\begin{abstract}
The detection of moving objects is the first step in video surveillance systems. But due to the challenging backgrounds such as illumination conditions, color saturation, and shadows, etc., the state of the art methods do not provide accurate segmentation using only a single camera. Recently, subspace learning model such as Robust Principal Component analysis (RPCA) shows a very nice framework towards object detection. But, RPCA presents the limitations of computational and memory issues due to the batch optimization methods, and hence it cannot process high dimensional data. Recent research on RPCA methods such as Online RPCA (OR-PCA) alleviates the traditional RPCA limitations. However, OR-PCA using only color or intensity features shows a weak performance specially when the background and foreground objects have a similar color or shadows appear in the background scene. To handle these challenges, this paper presents an extension of OR-PCA with the integration of depth and color information for robust background subtraction. Depth is less affected by shadows or background/foreground color saturation issues. However, the foreground object may not be detected when it is far from the camera field as depth is less useful without color information. We show that the OR-PCA including spatiotemporal constraints provides accurate segmentation with the utilization of both color and depth features. Experimental evaluations on a well-defined benchmark dataset with other methods demonstrate that our proposed technique is a top performer using color and range information.
\end{abstract}

Keywords-OR-PCA, Background subtraction, Disparity, Color features.

\section{INTRODUCTION}

Accurate and precise detection of moving objects from video sequence is the first step in many computer vision and image processing applications. This pre-processing step consists of separating moving objects called "foreground" from the static scene called "background". However, it becomes really hard task when the scene has sudden illumination change or geometrical changes such as waving trees, water surfaces, etc. [1]

Many algorithms have been developed to tackle the challenging problems in the background subtraction (also known as foreground detection) [1]-[3]. Among them, Robust Principal Component Analysis (RPCA) based approach shows a very nice framework for object detection. The background sequence is modeled by low-dimensional subspace called low-rank matrix and sparse error constitutes the foreground objects. Fig. 1 shows an example of moving object segmentation using RPCA based approach.

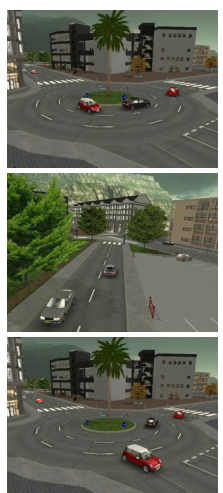

(a)

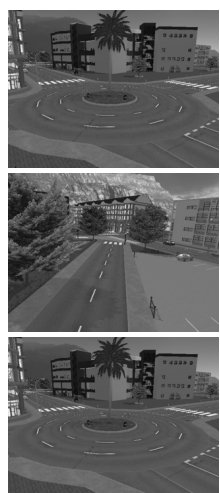

(b)

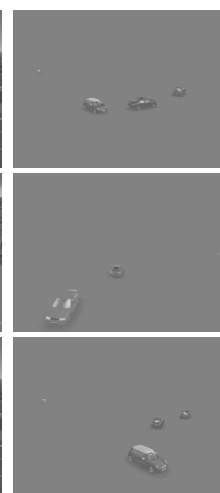

(c)

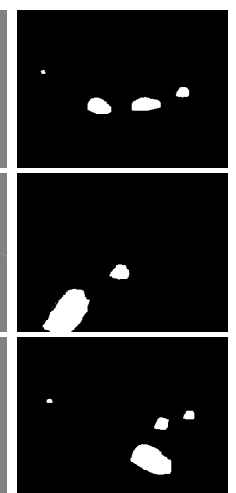

(d)
Fig. 1: A rotary sequence of BMC [4] dataset. From left to right: (a) input, (b) low-rank, (c) sparse, and (d) foreground mask.

Although RPCA for background subtraction attracts a lot of attention, but it faces some limitations. First, the algorithm includes batch optimization. In order to decompose an input image $A$ into low-rank matrix $L$ and sparse component $S$, a chunk of samples are required to store in memory. As a result, it suffers from huge memory usage and high computational cost. Second, there is no RPCA based approach which uses additional features such as edges, texture or depth information, as well as pixel intensity for background modeling, because it causes much more memory usages. Therefore, RPCA based scheme is not suitable for practical background subtraction systems. However, the most recent work on RPCA such as Online RPCA (OR-PCA) solves most of the traditional RPCA problems.

Online Robust Principal Component Analysis (ORPCA) [5] process one frame per time instance via stochastic optimization, provides a very interesting solution of RPCA based scheme. In [1] and [2], OR-PCA is modified to be adapted for background/foreground separation via image decomposition with initialization scheme using Markov Random Field (MRF). However, only intensity features are considered in this work. Due to the tedious parameter setting, the system is not applicable for real-time processing. As OR-PCA [2] 
method is able to provide robust background subtraction under different well-known challenges such as sudden illumination changes, waving trees, water surface, etc. But using a single camera, the problem is still far away from being solved specially when the background/foreground objects contain similar features and shadows appear in a background sequence.

In this paper, we present depth extended OR-PCA (DEORPCA) with spatiotemporal constraints for robust background subtraction using binocular cameras and stereo algorithms to compute the disparity image from pair of images. Depth information using three disparity estimation methods such as phase [6], variational [7] and semi-global block matching (SGBM) [8] based, with color features in OR-PCA produce accurate foreground segmentation. We briefly summarize our methodology here. First, the depth information is estimated from stereo pairs using three disparity estimation methods. Second, OR-PCA is applied on every color and depth frame to separate the low-rank and sparse component. Then the integration is performed on each separated low-rank and sparse matrix and a hard thresholding scheme is applied to obtain the initial foreground mask from integrated sparse component. Morever, spatiotemporal constraints such as MRF is applied on initial foreground mask to further improve the segmentation. In addition, a very nice comparison of foreground detection based on three types of disparity methods is presented.

The rest of this paper is organized as follows. In Section II, the related work is reviewed. Section III describes the proposed extended OR-PCA framework based on color and depth information. Experimental results are discussed in Section IV, and finally the conclusion and some future directions are shown in Section V.

\section{RELATED WORK}

Over the past few years, excellent methods have been proposed for background subtraction using subspace learning model [9]. Among them, Oliver et al. [10] are the first authors to model the background using Principal Components Analysis (PCA). The foreground detection is then achieved by thresholding the difference between the reconstructed background and input image. PCA provides a robust subspace learning model but it is not robust when the data is corrupted and outliers appear in the new subspace basis. In contrast, recent RPCA based approaches in [9] can tackle the problem of traditional PCA.

A remarkable improvements have been found on RPCA for background modeling. Excellent surveys on background modeling using RPCA can be found in [9]. For example, Candes et al. [11] proposed a robust convex optimization technique to address the PCA problems. Many batch optimization methods, such as Augmented Lagrangian Multiplier (ALM), Singular Value Thresholding (SVT) and Linearized Alternating Direction Method with an Adaptive Penalty (LADMAP) discussed in [9] solve the sub-optimization problem to separate the lowrank matrix and sparse error in each iteration under defined convergence criteria. These RPCA methods work in a batch optimization manner, as a result huge memory usage and high time complexity issues occur.

Therefore, Feng and $\mathrm{Xu}$ [5] recently proposed Online Robust-PCA (OR-PCA) algorithm which processes one chunk per time instance using stochastic approximations (no batch optimization is needed). A nuclear norm objective function is reformulated in this approach, and therefore all the samples are decoupled in optimization process for sparse error separation but no interesting results are observed in this work for background subtraction application. Therefore, Javed et al. [1] modified OR-PCA for background/foreground separation. Only intensity information via image decomposition including data-dependent initialization scheme is proposed in thier work. As a result the method is robust to some extent but it gets fail under highly dynamic background scenes.

Therefore, Javed et.al [2] further enhanced the OR-PCA using intensity features with continuous constraints such as Markov Random Field (MRF) to address the highly dynamic background scenes. A number of encouraging results have been observed in [2]. But annoying paramter tunning and lack of useful features are the main drawback in their approach. In [12], multiple features based OR-PCA for robust background subtraction is proposed Nine different features are used in this work and foreground detection is acheived using dynamic feature selection scheme based on feature similarity among low-rank features. Although the method is very robust for background subtraction but due to the computational complexity issues the method is not applicable for real-time applications.

There are significant work already being studied in [13][16] on background subtraction using disparity information. For example, Ivanov et.al [13] proposed an approach based on the use of range information to warp on image pair with the other one. This approach is not based on background subtraction method and the foreground detection is required if the color and brightness between corresponding points do not match.

In [14], Kolgomorov et.al proposed two algorithms for bilayer detection using fusion of stereo and color information based on the live background substitution. The foreground objects are detected using background subtraction on stereo pairs. Schiller [15] also proposed color based background subtraction with fusing depth information obtained using lowresolution Time-Of-Flight camera. Due to its low resolution, the method is not robust at objects boundaries.

In [16], a codebook algorithm is extended with fusing depth information. A very interesting study is presented for object segmentation on three types of disparity estimation algorithms as diuscussed above.

In this work, our main contributions can be summarized as follows: since RPCA based schemes either works in online or batch optimization manners using only a single camera, therefore we present an extension of OR-PCA [2] for binocular camera case for robust background subtraction. The three different types of disparity images are computed and OR-PCA is applied on each color and disparity image to get the sparse component. OR-PCA with disparity information provides an accurate segmentation on a wide range of background scenes.

\section{Methodology}

In this section, DEOR-PCA (depth extended OR-PCA) is discussed in detail. Our methodology consists of several components which are described as a system diagram in Fig. 2. 


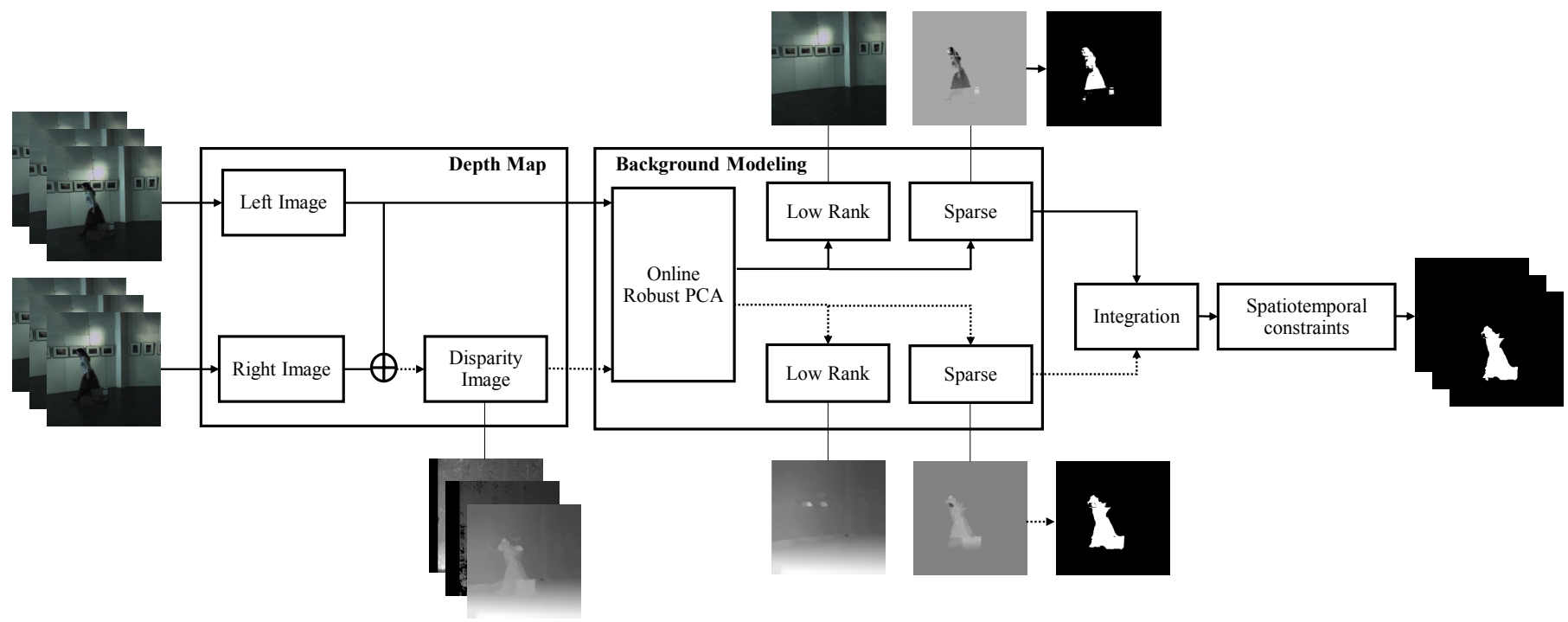

Fig. 2: Overview of our background modeling scheme.

Our methodology consists of four main stages: disparity estimation, background modeling, integration and spatialtemporal constraints. Initially, the range information is obtained using disparity estimation algorithms on a set of stereo pairs. Then, OR-PCA is applied to each of left and disparity images to model the background, separately. Next the integration is performed, which combines low-rank and sparse components obtained via OR-PCA to recover the background model and foreground mask from each image. The reconstructed sparse matrix is then thresholded to get the binary foreground mask. Finally, the spatiotemporal constraints such as continuous MRF is applied to remove most of the noise in the foreground mask due to depth information. In the following sections, we will describe each module in detail.

\section{A. Disparity Estimation}

In the first stage, three types of depth images are computed using variational [7], phase [6] and SGBM [8] based disparity algorithms. In this work, the disparity information is already provided as we use the benchmark dataset [17]. However in real-time processing, the disparity map can be obtained in the same way. Fig. 3 shows an example of input with three types of depth images taken from the dataset described in [16]. These depth images are computed based on color correspondence, therefore the noise can effect segmentation results from the events such as flickering of lights, and uniform regions, etc.

Since the range is less effected, when shadows appear in a background scene or foreground objects contains similar color features as background. But when the disparity is less useful e.g., foreground objects are not very close from the camera, the method may not produce correct results. Therefore both color and disparity features are useful. Overall, DEOR-PCA performs accurate foreground segmentation using variational based disparity which is discussed in the later section.

\section{B. Background Modeling}

Online Robust PCA [5] is applied on each depth and color frame. OR-PCA decomposes the nuclear norm of the objective function of the traditional PCP algorithms into an explicit product of two low-rank matrices, i.e., basis and coefficient. Thus, OR-PCA can be formulated as

$$
\begin{aligned}
\min _{L \in \Re d \times p, R \in \Re p \times r}, E & \left\{\frac{1}{2}\left\|D-L R^{T}-E\right\|_{F}^{2}\right. \\
& \left.+\frac{\lambda_{1}}{2}\left(\|L\|_{F}^{2}+\|R\|_{F}^{2}\right)+\lambda_{2}\|E\|_{1}\right\},
\end{aligned}
$$

where $D$ is an input data of any size (one sample), $d$ is the number of pixels with three color features, e.g. (width $\times$ height $\times 3$ ), $L$ is a basis of each individual color channel, $R$ is a coefficient and $E$ is a sparse error. $\lambda_{1}$ controls the basis and coefficients for low-rank matrix, whereas $\lambda_{2}$ controls the sparsity pattern, which can be tunned according to video analysis. In addition, basis and coefficient depend on the value of rank $r$, which is tunned carefully to speed up the stochastic optimization process.

In particular, the OR-PCA optimization consists of two iterative updating components. First, every incoming range and disparity image is projected onto current initialized basis $L$ and we separate the sparse noise component, which includes the outliers contamination. Then, the basis $L$ is updated with a

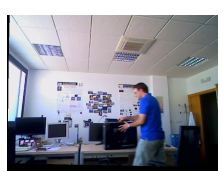

(a)

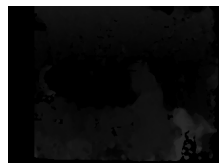

(b)

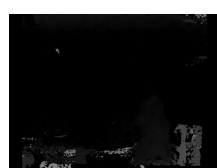

(c)

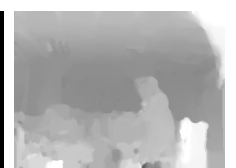

(d)
Fig. 3: Sample LCD Screen sequence. From left to right: (a) input, (b) Phase, (c) SGBM, and (d) variational disparity. 
new color and depth image individually. More details can be found in [5].

OR-PCA is applied to each color and disparity image using Eqn. 1 to get the low-rank and sparse matrix for each component. The background sequence for each image is then modeled by a multiple of basis $L$ and its coefficient $R$ e.g., $X=L R^{t}$, whereas the sparse component $E$ for each image constitutes the foreground objects.

\section{Integration}

The low-rank and sparse components are obtained from each feature after applying OR-PCA. Color and range lowrank and sparse components are integrated in this step. We use same parameters setting for OR-PCA in Eq.(1) for each feature. $\lambda_{1}$ is considered as a constant 0.01 for both images. $\lambda_{2}$ and rank $r$ for depth information, whereas $\lambda_{2}^{\prime}$ and rank $r^{\prime}$ for color frame are selected according to background scene, for obtaining enough sparsity pattern for each image.

Since disparity sparse component provides more information in background scene, therefore $\lambda_{2}$ must be the same as $\lambda_{2}^{\prime}$. After integrating sparse components of each image by adding individual features, the binary foreground mask $f$ is then obtained by thresholding the integrated sparse component. A hard thresholding scheme is applied to get the initial foreground map.

At this stage, the background subtraction scheme is good enough using variational based disparity information to deal with static and some small background dynamics as shown in Fig. 4 (d). However as illustrated in Fig. 4 (b) and (c), the integrated color and phase as well as SGBM based range information using OR-PCA produces false alarms. As these methods are very sensitive against noise or flickering of lights, which effect the quality of depth map. In addition, as discussed above color features do not provide enough information to detect objects which contain similar features as background but depth is less effected and hence our method provides an accurate segmentation as shown in Fig. 4 (d).

We use the parameters in Eqn. 1 as $r=r^{\prime}=1$, and $\lambda_{2}=\lambda_{2}^{\prime}=1 / \sqrt{\max (\text { width, height) }}$ for both images, which speed up the stochastic process for image decomposition. OR-PCA on color image with the integration of phase and SGBM based disparity produces noise without spatiotemporal constraints. As a result, a large number of false alarms are generated, which is not useful for visual surveillance system. Therefore, we have employed a spatiotemporal information in the foreground mask such as MRF, which improves the quality of foreground detection and alleviates most of the noise in depth mask.

\section{Spatiotemporal Constraints}

The initial foreground labeling is not optimal and therefore it can be improved with spatiotemporal constraints. In this paper, we utilize an MRF to optimize the initial labeling field. The MRF is a set of random variables having a Markov property described by an undirected graph.

Let us consider the foreground image $f$ as a set of pixels $\mathcal{P}$ and a set of labels $\mathcal{L}=\{0,1\}$, such that
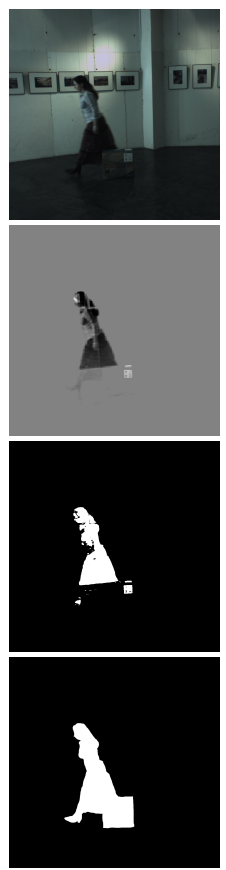

(a)
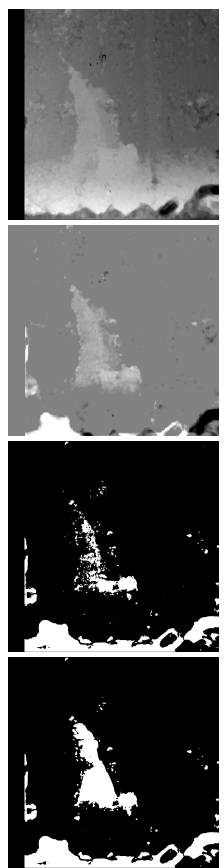

(b)
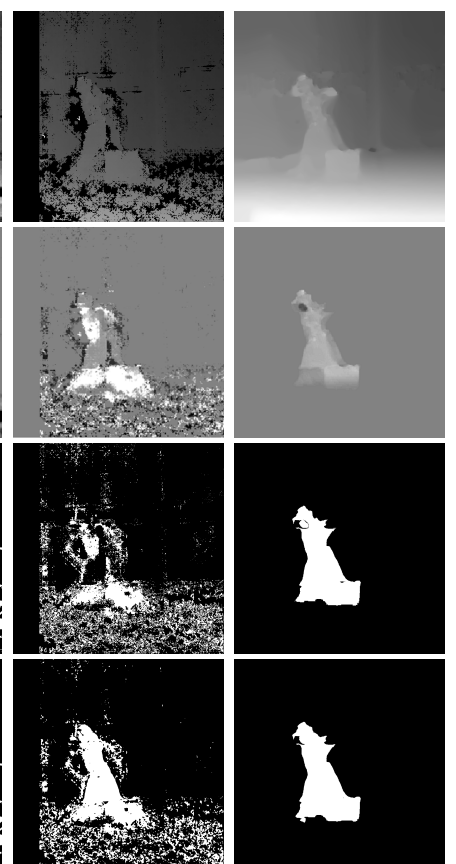

(c)

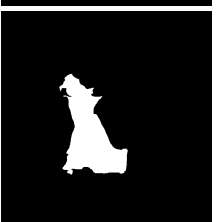

(d)
Fig. 4: Suitecase sequence. Input with disparity images, sparse component, thresholded mask, and ground truth with integrated foreground mask are shown in each of rows.

$$
f_{i}= \begin{cases}0, & \text { if } p \text { belongs to background } \\ 1, & \text { if } p \text { belongs to foreground. }\end{cases}
$$

The goal is to find a labeling $f$ which minimizes the energy function:

$$
E(f)=\sum_{i \in K} U_{i}\left(f_{i}\right)+\sum_{i, j \in \mathcal{N}} V_{i, j}\left(f_{i}, f_{j}\right),
$$

where $\mathcal{N} \subset \mathcal{P} \times \mathcal{P}$ is a neighborhood system on pixels, the function $U_{i}\left(f_{i}\right)$ penalizes or assigns pixel belonging to foreground and $V_{i, j}\left(f_{i}, f_{j}\right)$ controls the continuous degree of foreground or measures the cost of assigning the labels to the adajacent pixels.

The basic idea of graph cuts is to construct a directed graph $\mathcal{G}=(\mathcal{V}, \mathcal{E})$, where the vertices $\mathcal{V}$ stands for all pixels in image and edges $\mathcal{E}$ denotes spatially neighboring pixels having nonnegative weights that has two special vertices (terminals), namely, the source $s$ and the sink $t$.

In this work, we have used the gco-v3.0 library [18] for optimizing multi-label energies via the $\alpha$-expansion and $\alpha$ - $\beta$ swap algorithms. It supports energies with any combination of unary, pairwise, and label cost terms.

\section{EXPERIMENTAL EVALUATIONS}

In this section we present a set of both qualitative and quantitative experiments on a real video sequence benchmark dataset [16]. Our goal is to show that only color information is not enough for background subtraction and it can be enhance with disparity images. 


\section{A. Qualitative Analysis}

Due to the space limitations, some specific qualitative results are presented and we compare our method with other well known state of the art methods, e.g 4D Mixture of Gaussians (MOG4D) [15], PBAS [19], and Depth Extended Codebook [16] (DECB). In addition, we have also evaluated our method DEOR-PCA with three disparity based estiamtion algroithms such as DEOR-PCA variational, DEOR-PCA phase, and DE-ORPCA SGBM based foreground detection.

In [16], every sequence presents a major challenge such as suitecase sequence presents the low lighting and color saturation problem, crossing sequence contains foreground objects in and out of camera field where detection is useful based on color features. Similary, flickering of light challenge is presented in $L C D$ screen, shadows are appeared in $L a b$ door sequence. Fig. 5, 6 and 7 show the visual results of our proposed technique.

\section{B. Quantitative Analysis}

Quantitative results are also presented for the benchmark dataset. Our algorithm is implemented in Matlab R2013a with $3.40 \mathrm{GHz}$ Intel core i5 processor with 4 GB RAM. Additionally, $5 \times 5$ median filtering is applied as a post-processing step on binary foreground mask. The dataset contains four video sequences with hand segmented ground truth available for specific scene to compute the metrics. Cameras are calibrated and each pair of images is rectified for accurate disparity computation. The suitecase and crossing sequence contains an image size of $1,024 \times 1,024$ whereas $L C D$ screen and $L a b$ door video have a frame size of $640 \times 480$.

The F-measure score is computed for each specific sequence with its hand segmented ground truth data and the average $F$-measure score is provided in each case. The $F$ measure is given as

$$
F_{\text {measure }}=\frac{2 \times \text { Recall } \times \text { Precision }}{\text { Recall }+ \text { Precision }},
$$

where Recall and Precision are computed based on true positives, false positives and false negatives, respectively. Table. I shows a brief analysis of quantitive results in detail. Computational complexity is also observed during experimental evaluations. The computational time is recorded in CPU time as hh:mm::ss for 100 frames as shown in Table. II.

\section{Discussion}

In suitecase sequence, Fig. 5 provides three types of visual segmentation results. In this scenario, the suitcase contains similar color features with the background scene, therefore the detection is based mainly on depth information, and variational as well as phase based disparity approach provides good results as compared to SGBM as shown in Fig. 5 (f), (g), and (h) respectively. The noise in depth images is due to camera noise or disparity estimation algorithms.

Similarly, the crossing sequence in Fig. 6 presents two major difficulties. First, foreground objects are in and out of the camera field and the objects are detected based on color features. Second, due to color saturation of foregorund objects and shadows, the color feature is less important for detection.

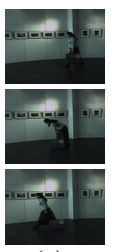

(a)

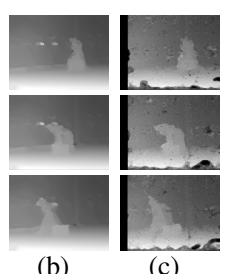

(b)

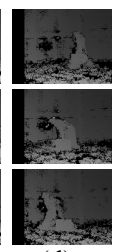

(d)

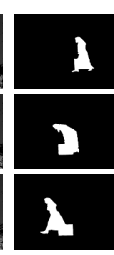

(e)

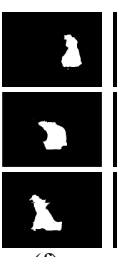

(f)

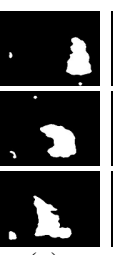

(g)

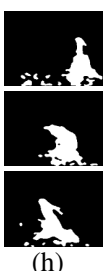

(h)
Fig. 5: Suitecase sequence. From left to right: (a) input, (b) variational, (c) phase, (d) SGBM, (e) ground truth, (f) DEOR-PCA variational, (g) DEOR-PCA phase, and (h) DEOR-PCA SGBM based foreground mask.

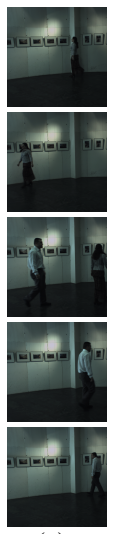

(a)

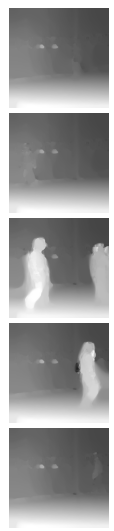

(b)

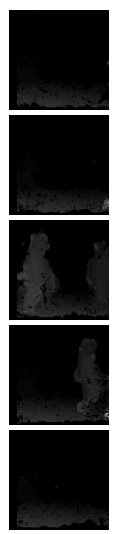

(c)

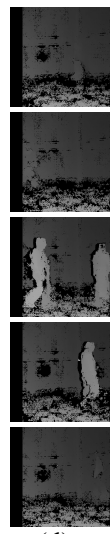

(d)

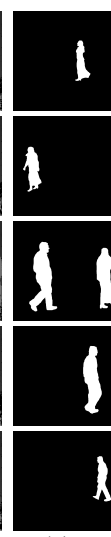

(e)

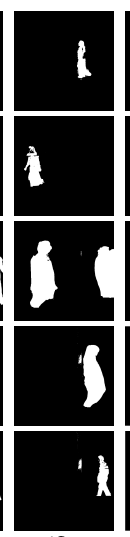

(f)

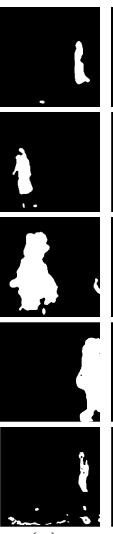

(g)

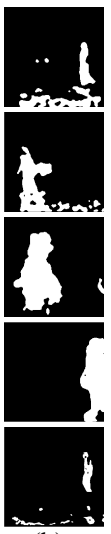

(h)
Fig. 6: Crossing sequence. From left to right: (a) input, (b) variational, (c) phase, (d) SGBM, (e) ground truth, (f) DEOR-PCA variational, (g) DEOR-PCA phase, and (h) DEOR-PCA SGBM based foreground mask.

As compared to the suitcase category, the detection is based on both color and depth information for crossing sequence and variational based disparity approach gives accurate foreground segmentation as depicted in Fig. 6 (f).

Furthermore, the lab door sequence has a flickering of light and the object is close and far from the camera field. Both features are important and variational based disparity information improves the accuracy as compares to other two approaches as shown in Fig. 7 and Table. I.

\begin{tabular}{|c|c|c|c|c|c|}
\hline Method & Suitecase & Crossing & LCD screen & Lab door & Avr. \\
\hline MOG4D-phase & 0.412 & 0.658 & 0.781 & 0.492 & 0.585 \\
\hline MOG4D-SGBM & 0.433 & 0.457 & 0.687 & 0.570 & 0.536 \\
\hline MOG4D-Var & 0.762 & 0.778 & 0.625 & 0.431 & 0.649 \\
\hline PBAS & 0.565 & 0.853 & 0.708 & 0.725 & 0.712 \\
\hline DECB-phase & 0.499 & 0.636 & 0.691 & 0.658 & 0.621 \\
\hline DECB-SGBM & 0.790 & 0.851 & $\mathbf{0 . 8 3 2}$ & 0.691 & 0.791 \\
\hline DECB-Var & 0.766 & 0.780 & 0.803 & 0.548 & 0.724 \\
\hline DEOR-PCA-phase & 0.431 & 0.620 & 0.684 & 0.5467 & 0.674 \\
\hline DEOR-PCA-SGBM & 0.413 & 0.416 & 0.668 & 0.572 & 0.617 \\
\hline DEOR-PCA-Var & $\mathbf{0 . 8 2 6}$ & $\mathbf{0 . 9 0 6}$ & 0.764 & $\mathbf{0 . 7 8 0}$ & $\mathbf{0 . 8 1 9}$ \\
\hline
\end{tabular}

TABLE I: Quantitative comparison of F-measure score with other state of the art methods of overall dataset. Bold values represent the best $F$-measure whereas the bold red value indicates the best average $F$-measure on all sequences. 


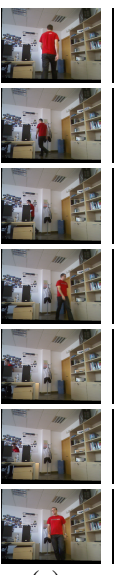

(a)

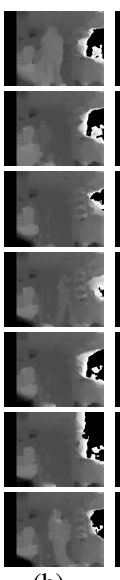

(b)

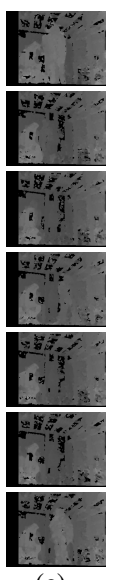

(c)

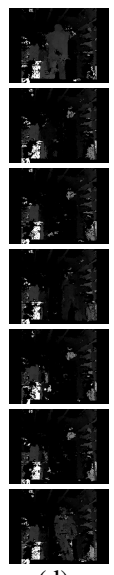

(d)

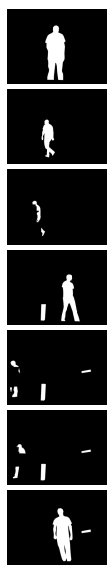

(e)

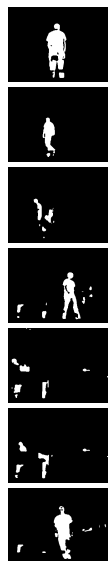

(f)

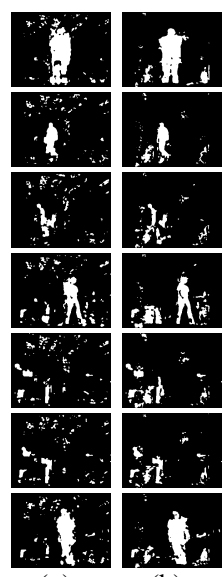

(g) (h)
Fig. 7: Lab door sequence. From left to right: (a) input, (b) variational, (c) phase, (d) SGBM, (e) ground truth, (f) DEOR-PCA variational, (g) DEOR-PCA phase, and (h) DEOR-PCA SGBM based foreground mask.

\begin{tabular}{|c|c|c|c|c|}
\hline Method & $1024 \times 1024$ & $512 \times 512$ & $640 \times 480$ & $320 \times 240$ \\
\hline DEOR-PCA & $00: 01: 12$ & $00: 00: 24$ & $00: 00: 27$ & $00: 00: 09$ \\
\hline
\end{tabular}

TABLE II: Time complexity according to different image resolution.

\section{CONClusion}

In this paper, a robust background modeling method against challenging background scenes is presented using both color and disparity information on OR-PCA with spatiotemporal constraints. Our methodology is robust against different background dynamics. We applied three types of range information on OR-PCA. In addition, experimental evaluations and comparisons with other state of the art methods show the effectiveness and robustness of our proposed scheme with different type of range information, which demonstrate that subspace learning model shows a very nice potential for background/foreground separation using binocular cameras.

First, the variational based range information provides an accurate foreground segmentation, however SGBM and phase estimation algorithms make a noise sometimes and hence the accuracy is reduced. Second, only one dataset is available, which is tested using OR-PCA. Therefore, our future work will focus more on brief analysis of each depth information with fusion technique to make the algrotihm more robust and rigorous experimental results will be presented in more details.

\section{ACKNOWLEDGMENT}

This work is supported by the World Class 300 project, Development of HD video/network-based video surveillance system(10040370), funded by the Ministry of Trade, Industry, and Energy (MOTIE), Korea.

\section{REFERENCES}

[1] S. Javed, S. H. Oh, J. Heo, and S. K. Jung, "Robust Background Subtraction via Online Robust PCA using image Decomposition," in Proceedings of the 2014 Research in Adaptive and Convergent Systems, 2014, pp. 90-96.
[2] S. Javed, S. OH, S. Andrews, T. Bouwmans, and S. K. Jung, "ORPCA with MRF for Robust Foreground Detection in Highly Dynamic Backgrounds," in 12th Asian Conference on Computer Vision (ACCV), 2014, 2014, p. To appear.

[3] S. H. Oh, S. Javed, and S. K. Jung, "Foreground object detection and tracking for visual surveillance system: A hybrid approach," in Frontiers of Information Technology (FIT), 2013 11th International Conference on, Dec, pp. 13-18.

[4] A. Vacavant, T. Chateau, A. Wilhelm, and L. Lequièvre, "A benchmark dataset for outdoor foreground/background extraction," in Computer Vision-ACCV 2012 Workshops. Springer, 2013, pp. 291-300.

[5] J. Feng, H. Xu, and S. Yan, "Online Robust PCA via stochastic optimization," in Advances in Neural Information Processing Systems, 2013, pp. 404-412.

[6] M. Tomasi, M. Vanegas, F. Barranco, J. Daz, and E. Ros, "Massive parallel-hardware architecture for multiscale stereo, optical flow and image-structure computation," Circuits and Systems for Video Technology, IEEE Transactions on, vol. 22, no. 2, pp. 282-294, 2012.

[7] J. Ralli, J. Díaz, and E. Ros, "Spatial and temporal constraints in variational correspondence methods," Machine vision and applications, vol. 24, no. 2, pp. 275-287, 2013.

[8] H. Hirschmuller, "Stereo processing by semiglobal matching and mutual information," Pattern Analysis and Machine Intelligence, IEEE Transactions on, vol. 30, no. 2, pp. 328-341, 2008.

[9] T. Bouwmans and E. H. Zahzah, "Robust PCA via Principal Component Pursuit: A review for a comparative evaluation in video surveillance," Computer Vision and Image Understanding, pp. 22-34, 2014.

[10] N. M. Oliver, B. Rosario, and A. P. Pentland, "A bayesian computer vision system for modeling human interactions," Pattern Analysis and Machine Intelligence, IEEE Transactions on, vol. 22, no. 8, pp. 831843, 2000.

[11] E. J. Candès, X. Li, Y. Ma, and J. Wright, "Robust Principal Component Analysis?" Journal of the ACM (JACM), vol. 58, no. 3, p. 11, 2011.

[12] S. Javed, S. Andrews, T. Bouwmans, and S. K. Jung, "OR-PCA with Dynamic Feature Selection for Robust Background Subtraction," in The 30th ACM/SIGAPP Symposium on Applied Computing, 2015, p. To appear.

[13] Y. Ivanov, A. Bobick, and J. Liu, "Fast lighting independent background subtraction," International Journal of Computer Vision, vol. 37, no. 2, pp. 199-207, 2000.

[14] V. Kolmogorov, A. Criminisi, A. Blake, G. Cross, and C. Rother, "Bi-layer segmentation of binocular stereo video," in Computer Vision and Pattern Recognition, 2005. CVPR 2005. IEEE Computer Society Conference on, vol. 2, 2005, pp. 407-414.

[15] I. Schiller and R. Koch, "Improved video segmentation by adaptive combination of depth keying and mixture-of-gaussians," in Image Analysis, 2011, pp. 59-68.

[16] E. J. Fernandez-Sanchez, L. Rubio, J. Diaz, and E. Ros, "Background subtraction model based on color and depth cues," Machine Vision and Applications, pp. 1-15, 2013.

[17] [Online]. Available: http://atcproyectos.ugr.es/mvision

[18] [Online]. Available: http://vision.csd.uwo.ca/code/

[19] M. Hofmann, P. Tiefenbacher, and G. Rigoll, "Background segmentation with feedback: The pixel-based adaptive segmenter," in Computer Vision and Pattern Recognition Workshops (CVPRW), 2012 IEEE Computer Society Conference on, 2012, pp. 38-43. 